Journal of Psychopharmacology 13(2) (1999) 136-143

(C)1999 British Association for Psychopharmacology (ISSN 0269-88I1)

SAGE Publications, London, Thousand Oaks, CA and New Delhi

0269-8811 [199905] 13:2; 136-143; 008435

\title{
Longitudinal patterns of antidepressant prescribing in primary care in the UK: comparison with treatment guidelines
}

\author{
Rodney L. Dunn ${ }^{1}$, John M. Donoghue ${ }^{2}$, Ronald J. Ozminkowski³ ${ }^{3}$ Deborah Stephenson ${ }^{4}$ \\ and Timothy R. Hylan ${ }^{5}$ \\ ${ }^{1}$ University of Michigan, Ann Arbor, MI, USA, ${ }^{2}$ PCS Health, Liverpool, UK, ${ }^{3}$ The MEDSTAT Group, Ann Arbor, MI, USA, ${ }^{4}$ Lilly Industries, \\ Basingstoke, UK and ${ }^{5}$ Eli Lilly and Company, Indianapolis, IN, USA.
}

The objective of this study was to determine whether patients beginning therapy on the most common tricyclic antidepressants (TCAs) and selective serotonin reuptake inhibitors (SSRIs) differed in their likelihood of having antidepressant treatment that was consistent with recommended treatment guidelines in the UK. An analytical file constructed from a large general practitioner medical records database (DIN-LINK) from the UK for the years 1992-97 was constructed. A total of 16204 patients with a new episode of antidepressant therapy who initiated therapy on one of the most often prescribed TCAs (amitriptyline, dothiepin, imipramine and lofepramine) or SSRIs (fluoxetine, paroxetine and sertraline) were analysed. A dichotomous measure was defined to indicate whether subjects were prescribed at least 120 days of antidepressant therapy at an adequate average daily dose within the first 6 months after initiation of therapy. Only $6.0 \%$ of patients initiating therapy on a TCA and $32.9 \%$ of patients initiating therapy on a SSRI were prescribed antidepressant treatment that was consistent with treatment guidelines. After controlling for observable characteristics, patients who initiated therapy on a SSRI were much more likely (odds ratio $=7.473, p<0.001$ ) to have a prescribed average daily dose and duration consistent with recommended treatment guidelines within the first 6 months of initiating therapy than were patients who initiated therapy on a TCA. These findings suggest that initial antidepressant selection is an important determinant of whether the subsequent course of treatment is consistent with current national guidelines for the treatment of depression in the UK.

Key words: antidepressants; depression; drug prescribing patterns; selective serotonin reuptake inhibitors; tricyclic antidepressants

\section{Introduction}

Antidepressant dose and duration are two important factors in achieving the goals of depression treatment which include not only symptom resolution, but also restoration of patients' functioning and prevention of relapse or recurrent episodes. Consensus guidelines have been issued by the British Association for Psychopharmacology (British Association for Psychopharmacology, 1993) and the Royal College of Psychiatrists (Royal College of Psychiatrists, 1992) for the management of depression with antidepressants. For treatment to be effective, they recommend that antidepressants should be prescribed at a dose shown by clinical trials to be effective in treating depression, and continued for at least 4 months beyond initial symptom resolution. These guidelines are broadly consistent with other consensus groups' recommendations (World Health Organization Mental Health Collaborating Centres, 1989; Agency for Health Care Policy Research, 1993).

Most clinical trials of different antidepressants generally show equal efficacy (Effective Health Care Bulletin, 1993;
Anderson and Thomenson, 1994; Song et al., 1994). However, studies of antidepressant use in clinical practice ('naturalistic' studies) have shown that most patients who initiate therapy on a tricyclic antidepressant (TCA) in primary care in the UK begin at suboptimal doses and remain there (Donoghue and Tylee, 1996; Donoghue et al., 1996; Martin et al., 1997). In addition, studies have also shown that most patients beginning treatment with a TCA are less likely than patients on selective serotonin reuptake inhibitors (SSRIs) to refill their prescriptions and often discontinue therapy by the third month (MacDonald, 1997).

Naturalistic, observational studies of antidepressant use in clinical practice can complement the findings from controlled trials. A feature of observational studies is that the results are more generalizable to broader populations than those considered in controlled clinical trials due to the lack of study conditions or protocol restrictions. However, unlike randomized controlled clinical trials, observational studies are subject to the influence of confounding factors. Previous observational studies of antidepressant prescribing patterns in primary care in the UK have been limited by their cross-sectional design 
(Donoghue, 1995, 1996; Donoghue and Tylee, 1996; Donoghue et al., 1996). In addition, the only naturalistic, longitudinal study of antidepressant prescribing patterns in the UK to date did not address the influence of other observed patient or prescriber characteristics on prescribing patterns (MacDonald, 1997). Thus, it was unclear whether the differences in observed prescribing patterns were due to the drugs or to other factors that influence both drug selection and prescribing.

The purpose of this study was to assess how the prescribing patterns of different antidepressants in the first 6 months following initiation of treatment compare with recommended depression treatment guidelines in the UK. We considered a sample of patients in primary care who had a depressionrelated diagnosis at the initiation of therapy. All patients in this study initiated therapy on one of the most commonly prescribed TCAs (amitriptyline, dothiepin, imipramine and lofepramine) or SSRIs (fluoxetine, paroxetine and sertraline), which represented over $97 \%$ of all antidepressant prescribing in primary care in this study. Unlike earlier studies, this one considers a longer period of prescribing data (1992-97), constructs and analyses patients' episodes of antidepressant use, and uses logistic regression analysis to control for the influence of other observed factors.

\section{Methods}

\section{Data}

The analysis file used in this study was constructed from the 1992-97 panels of the Doctors' Independent Network (DINLINK), which is maintained by CompuFile Ltd. (Woking, UK). DIN-LINK is a system of comprehensive and standardized medical and prescription records for approximately 750000 patients from 100 general practices in the UK that use AAH Meditel practice software. A 6-month antidepressantfree period prior to the index date was used to ensure that antidepressant prescribing was not part of an earlier episode. Episodes of antidepressant therapy were then constructed using an intent-to-treat basis. In an intent-to-treat design, patients' subsequent experience in the study period is attributed back to the original TCA or SSRI selected, regardless of subsequent use. For example, if a patient begins on a TCA and then switches to a SSRI or another antidepressant, the patient remains in the TCA category for the analysis. An intent-to-treat analysis is consistent with examining the consequences of initial antidepressant selection (Simon et al., 1996).

The 'index study' antidepressant was one of the four study TCAs or three SSRIs on which the patient began therapy. The index date was defined as the date on which the first prescription for the study antidepressant was issued and was accompanied by one of five depression-related diagnoses (as recorded in the medical record, including endogenous depression, reactive depression, depressed, on examination depressed or depression not otherwise specified) within a 30-day period around the index date. These diagnoses are consistent with those used and validated in earlier analyses of the DIN-LINK data (Donoghue, 1996; Donoghue and Tylee, 1996). General practitioners use all of these terms to describe depression in primary care in the UK, and do not use them consistently to differentiate between different manifestations of depression.

Patients were excluded if they: (1) were unable to be followed for at least 180 days before the index date; (2) did not have 180 days of continuous health care coverage in the same general practitioner practice after the index date; (3) were prescribed more than one index study antidepressant at the index date; (4) had antidepressant use in the 180-day prior period; (5) had multiple records of the study antidepressant on the index date; or (6) were less than 18 years of age at the index date. After the exclusions, the analytical file included 16204 patients representing 99 general practices.

\section{Statistical analysis}

A logistic regression model was used to determine the effect of drug class on antidepressant prescribing patterns. The dependent variable was defined as a dichotomous variable indicating whether or not the patient was prescribed antidepressant prescriptions at recommended average daily doses for an adequate duration.

Challenges in defining patterns of antidepressant prescribing with retrospective data are well-known (Simon et al., 1993; Fairman, 1997). Even with dates of prescriptions, it is not possible to know exactly when patients finished the contents of one prescription or started on another. Patients may take additional daily doses, miss doses during therapy, or refill prescriptions other than the day the supply of the previous prescription has been exhausted. Any measure of prescribing outcomes will involve compromises given the nature of the data. Adequate duration was met if the combined expected duration of all prescriptions was at least 120 days of the first 180 days beginning with initiation of therapy. This definition allowed for no more than one 30-day gap between antidepressant prescriptions in the first 6 months. Recommended daily dosages used were $20 \mathrm{mg}$ for paroxetine, fluoxetine, and citalopram, $50 \mathrm{mg}$ for sertraline, $75 \mathrm{mg}$ for venlafaxine, $100 \mathrm{mg}$ for nefazadone, $125 \mathrm{mg}$ for amitriptyline, amoxapine, clomipramine, desipramine, dothiepin, doxepin, imipramine, and maprotiline and $140 \mathrm{mg}$ for lofepramine. Thus, at least 120 days of antidepressant therapy at recommended daily doses within the first 6 months after initiation of therapy was considered to be a reasonable estimation of treatment consistent with guidelines in the UK.

The main explanatory variable was a binary indicator of SSRI use, with TCAs, the most common antidepressants, as the reference group. Controlling for other factors in the regression, this variable enables a comparison of the odds of receiving adequate treatment for patients initiating therapy on an SSRI relative to patients initiating therapy on a TCA.

Other factors besides drug selection may also influence the prescribing patterns and were included in the analysis as additional explanatory variables. These factors include patient demographics (age and sex) and region of the country. Other variables included were related to the patient's comorbid conditions at the index date or in the prior period. First, four binary indicators for depression diagnosis at the index date were created (i.e. endogenous depression, reactive depression, depressed and on examination depressed, with depression not otherwise specified as the reference group). Second, binary 
indicator variables were created for the following conditions if they occurred in the 180-day period prior to initiation of therapy: cancer, chronic fatigue syndrome, coronary artery disease, stroke, diabetes and fibromyalgia. These are conditions commonly coexisting with depression (AHCPR, 1993). Third, several variables were created which represented counts of the following occurrences in the 180-day prior period: anxiolytic prescriptions, sedative-hypnotics prescriptions, general practitioner visits, general practitioner referrals, psychotherapy referrals, laboratory referrals, psychiatric accident and emergency admissions, psychiatric non-accident and emergency admissions, general accident and emergency admissions and general non-accident and emergency admissions. Fourth, in an attempt to control for concomitant psychiatric conditions, indicators were created for Psychiatric Diagnostic Groups (Ashcraft et al., 1989) which are based on the ICD-9 classification scheme. Psychiatric Diagnostic Groups were defined as a method of classifying major psychiatric conditions and are therefore analogous to the Major Diagnostic Categories used in the Diagnosis Related Groups classification system

Finally, an additional variable was included to reduce the effect of factors related to the general practice characteristics such as habitual antidepressant prescribing practices. This variable represents the probability that the general practitioners at the patient's general practice prescribed SSRIs and was measured by computing the percentage of the general practice's study antidepressant prescriptions which were for SSRIs during the study period. Physicians' practice patterns may be shaped by a number of influences, including previous experience with the product and the information physicians receive about the product and its use. Thus, unlike previous studies of antidepressant prescribing, it was possible to control for underlying differences in general practices that are likely to prescribe TCAs or SSRIs. In this way, differences in physicians' habitual prescribing practices, preferences or previous experiences with different classes of drugs are controlled for (Simon and Fishman, 1998).

A standard significance level $(p<0.05)$ was used for the analysis. The SAS package (SAS Institute, Cary, North Carolina, USA) was used to construct the dataset and perform the logistic regression analysis.

\section{Results}

Table 1 presents descriptive results of the variables used in the analysis. Of the 16204 patients in the sample, $62.0 \%$ $(n=10054)$ initiated therapy on one of the most often prescribed TCAs (amitriptyline, dothiepin, imipramine and lofepramine) while approximately $38.0 \%(n=6150)$ initiated therapy on one of the most often prescribed SSRIs (fluoxetine, paroxetine and sertraline). Before adjusting for other potential confounding variables, SSRI patients $(32.9 \%)$ were much more likely than TCA patients $(6.0 \%)$ to be prescribed at least 120 days of antidepressant therapy at the recommended average daily dosage in the first 6 months after starting therapy. The main reason for SSRI patients not achieving adequate use was fewer than 120 days of therapy within the first 6 months. The main reason for TCA patients not achieving adequate use was average daily doses below the recommended values for one or more prescriptions. Notably, only $9.0 \%$ of the TCA treated patients experienced upward dose titration.

The unadjusted odds ratio of having an adequate dose and duration of antidepressant was 7.68. After controlling for other observable variables through logistic regression analysis, the results showed that the odds of having an adequate dose and duration of antidepressant therapy was still over sevenfold higher for patients taking a SSRI than for TCA patients (odds ratio $=7.473, p<0.001$, Table 2). By including the other variables related to patients' demographics and comorbid conditions, these factors were controlled for in the analysis Although a number of these other variables were statistically significant individually, the adjusted odds ratio (7.47) and the unadjusted odds ratio (7.68) were similar.

These results were not sensitive to alternative specifications of the model and different definitions of an adequate prescribing pattern. An additional analysis was performed using $75 \mathrm{mg}$ as a minimum average daily dose for the TCAs (except lofepramine). In this case, $15.6 \%$ of patients who started on a TCA were found to have an adequate dose and duration, compared with $34.1 \%$ of patients who started on a SSRI. For this analysis, the multiple regression results indicated that the odds of having an adequate dose and duration were nearly threefold higher for SSRI patients compared with TCA patients (odds ratio $=2.825, p<0.001$ ).

Analysis of lofepramine patients $(n=2076)$ separately indicated that lofepramine patients achieve adequate dose and duration $18.3 \%$ of the time, compared with $2.8 \%$ for patients on the other TCAs. Removing lofepramine subjects from the logistic regression resulted in an odds ratio of 17.063 $(p<0.001)$ for the comparison of SSRIs with the older TCAs, compared with an odds ratio of 7.473 when the lofepramine patients were included with the other TCA patients. Analysis of SSRI patients relative to lofepramine patients only resulted in an odds ratio of $2.236(p<0.001)$.

\section{Discussion}

How antidepressants are prescribed in clinical practice could differ due to differences in convenience, tolerability, and a variety of complex interactions between patient, prescriber and health care service characteristics. Meta-analyses of controlled trials of antidepressants (Anderson and Tomenson, 1994; Montgomery et al., 1994) have found differences in drop-out rates due to side-effects with lower rates for SSRIs relative to TCAs. The SSRIs are also less toxic in overdose (Henry et al., 1995). Recent analyses have also found a perception among general practitioners of differences in tolerability between the TCAs and SSRIs (Martin et al., 1997). In addition, it is acknowledged that lofepramine does appear to be used differently in clinical practice owing to (at least perceived) differences in tolerability (Donoghue and Tylee, 1996). Thus, there is some evidence that antidepressant prescribing in clinical practice may be driven, in part, by the perception of tolerability differences across TCAs and SSRIs.

In this study, overall rates of prescribing of TCA and SSRI antidepressants in relation to treatment guidelines were low. 
Table 1 Descriptive statistics by index study antidepressant class

\begin{tabular}{|c|c|c|c|c|c|}
\hline & \multicolumn{2}{|c|}{$\begin{array}{l}\text { Selective serotonin reuptake inhibitor } \\
\text { patients }(n=6150)\end{array}$} & \multicolumn{2}{|c|}{$\begin{array}{l}\text { Tricyclic antidepressant patients } \\
\qquad(n=10054)\end{array}$} & \multirow[b]{2}{*}{$p$-values* } \\
\hline & $n$ & $\%$ & $n$ & $\%$ & \\
\hline Adequate Dose and Duration & & & & & 0.001 \\
\hline Yes & 2022 & $32.9 \%$ & 604 & $6.0 \%$ & \\
\hline $\begin{array}{l}\text { No- - recommended dose but not } \\
\text { recommended duration }\end{array}$ & 3701 & $60.2 \%$ & 1324 & $13.2 \%$ & \\
\hline $\begin{array}{l}\text { No - recommended duration but not } \\
\text { recommended dose }\end{array}$ & 135 & $2.2 \%$ & 2454 & $24.4 \%$ & \\
\hline $\begin{array}{l}\text { No-not recommended dose or } \\
\text { duration }\end{array}$ & 292 & $4.7 \%$ & 5672 & $56.4 \%$ & \\
\hline Index Depression Diagnosis & & & & & 0.001 \\
\hline Depression not otherwise specified & 2967 & $48.2 \%$ & 4816 & $47.9 \%$ & \\
\hline Endogenous depression & 1506 & $24.5 \%$ & 2395 & $23.8 \%$ & \\
\hline Reactive depression & 797 & $13.0 \%$ & 1576 & $15.7 \%$ & \\
\hline Depressed & 753 & $12.2 \%$ & 1124 & $11.2 \%$ & \\
\hline On examination - depressed & 127 & $2.1 \%$ & 143 & $1.4 \%$ & \\
\hline Location & & & & & 0.001 \\
\hline Southern England & 3026 & $49.2 \%$ & 4411 & $43.9 \%$ & \\
\hline Northern England and Scotland & 2648 & $43.1 \%$ & 5041 & $50.1 \%$ & \\
\hline Wales & 476 & $7.7 \%$ & 602 & $6.0 \%$ & \\
\hline Sex & & & & & 0.001 \\
\hline Male & 1647 & $26.8 \%$ & 2345 & $23.3 \%$ & \\
\hline \multirow[t]{2}{*}{ Female } & 4503 & $73.2 \%$ & 7709 & $76.7 \%$ & \\
\hline & Mean & SD & Mean & SD & \\
\hline Age & 45.224 & 17.631 & 48.576 & 17.933 & $<0.001$ \\
\hline \multicolumn{6}{|c|}{ Counts of medical occurrences in the 180 day prior period } \\
\hline Anxiolytic prescriptions & 0.107 & 0.748 & 0.119 & 0.853 & 0.598 \\
\hline Sedative-hypnotics prescriptions & 0.247 & 1.286 & 0.273 & 1.282 & 0.557 \\
\hline General practitioner visits & 6.840 & 6.076 & 6.691 & 5.782 & 0.432 \\
\hline General practitioner referrals & 1.041 & 2.470 & 0.954 & 2.295 & 0.011 \\
\hline Psychotherapy referrals & 0.094 & 0.570 & 0.068 & 0.535 & $<0.001$ \\
\hline Laboratory referrals & 0.078 & 0.726 & 0.103 & 0.757 & 0.004 \\
\hline Psychiatric accident and emergency admissions & 0.010 & 0.136 & 0.005 & 0.096 & $<0.001$ \\
\hline $\begin{array}{l}\text { Psychiatric non-accident and emergency } \\
\text { admissions }\end{array}$ & 0.005 & 0.137 & 0.001 & 0.028 & 0.001 \\
\hline General accident and emergency admissions & 0.058 & 0.492 & 0.050 & 0.394 & 0.223 \\
\hline $\begin{array}{l}\text { General non-accident and emergency } \\
\text { admissions }\end{array}$ & 0.136 & 0.721 & 0.119 & 0.637 & 0.255 \\
\hline \multicolumn{6}{|c|}{ Indicator of Psychiatric Diagnostic Group occurrence in the 180 day prior period } \\
\hline $\begin{array}{l}\text { Organic mental disorders and } \\
\text { alcohol use disorders }\end{array}$ & 0.009 & 0.096 & 0.007 & 0.081 & 0.065 \\
\hline Opioid and other substance use disorders & 0.005 & 0.067 & 0.005 & 0.068 & 0.912 \\
\hline Schizophrenic disorders & 0.002 & 0.046 & 0.001 & 0.030 & 0.041 \\
\hline Other psychotic disorders & 0.001 & 0.025 & 0.001 & 0.026 & 0.914 \\
\hline Bipolar disorders & 0.001 & 0.031 & 0.001 & 0.030 & 0.870 \\
\hline Major depressions & 0.160 & 0.367 & 0.147 & 0.354 & 0.023 \\
\hline Other specific and atypical affective disorders & 0.001 & 0.031 & 0.001 & 0.036 & 0.567 \\
\hline Post-traumatic stress disorders & 0.005 & 0.067 & 0.004 & 0.067 & 0.943 \\
\hline Anxiety disorders & 0.045 & 0.207 & 0.041 & 0.198 & 0.245 \\
\hline Personality disorders & 0.001 & 0.025 & 0.002 & 0.040 & 0.098 \\
\hline $\begin{array}{l}\text { Impulse control, adjustment disorders, other } \\
\text { mental }\end{array}$ & 0.039 & 0.194 & 0.038 & 0.192 & 0.840 \\
\hline \multicolumn{6}{|c|}{ Indicator of other medical conditions in the 180 day prior period } \\
\hline Chronic fatigue syndrome & 0.003 & 0.054 & 0.004 & 0.063 & 0.277 \\
\hline Cancer & 0.013 & 0.111 & 0.014 & 0.117 & 0.482 \\
\hline Coronary artery disease & 0.051 & 0.219 & 0.043 & 0.204 & 0.030 \\
\hline Stroke & 0.016 & 0.127 & 0.014 & 0.117 & 0.202 \\
\hline Diabetes & 0.021 & 0.144 & 0.023 & 0.150 & 0.510 \\
\hline Fibromyalgia & 0.005 & 0.073 & 0.006 & 0.080 & 0.423 \\
\hline \multicolumn{6}{|l|}{ General Practice Drug Class Preference } \\
\hline $\begin{array}{l}\text { Frequency of general practice selective } \\
\text { serotonin reuptake inhibitor prescriptions }\end{array}$ & 0.444 & 0.170 & 0.323 & 0.144 & $<0.001$ \\
\hline
\end{tabular}

${ }^{*} p$-values for use patterns, index depression diagnosis, location, and sex are based on chi-squared tests of independence. The $p$-value for age is based on an unequal variance $t$-test. All other $p$-values are based on the non-parametric Wilcoxon rank-sum test. 
Table 2 Odds ratios (confidence intervals) and $p$-values from a logistic regression model predicting the log odds of having received 120 days of therapy on the study antidepressant in the first 6 months following initiation of therapy at an adequate dosage

\begin{tabular}{|c|c|c|}
\hline Variable & Odds ratio & $p$-value \\
\hline \multicolumn{3}{|l|}{ Drug type chosen } \\
\hline $\begin{array}{l}\text { Indicator of selective serotonin reuptake inhibitor as study } \\
\text { antidepressant class }\end{array}$ & $7.473(6.752-8.272)$ & $<0.001$ \\
\hline \multicolumn{3}{|l|}{ Demographics } \\
\hline Age at index date & $1.004(1.001-1.007)$ & 0.005 \\
\hline Female indicator & $0.918(0.828-1.019)$ & 0.108 \\
\hline \multicolumn{3}{|l|}{ Location } \\
\hline Wales indicator & $0.708(0.580-0.864)$ & 0.001 \\
\hline Southern England indicator & $1.023(0.931-1.123)$ & 0.641 \\
\hline \multicolumn{3}{|l|}{ Index Depression Diagnosis } \\
\hline Endogenous depression & $1.192(1.069-1.329)$ & 0.002 \\
\hline Reactive depression & $0.855(0.737-0.991)$ & 0.037 \\
\hline Depressed & $0.737(0.632-0.861)$ & $<0.001$ \\
\hline On examination depressed & $0.718(0.502-1.027)$ & 0.070 \\
\hline \multicolumn{3}{|l|}{ Counts of medical occurrences in the 180 day prior period } \\
\hline Anxiolytic prescriptions & $1.005(0.951-1.062)$ & 0.866 \\
\hline Sedative-hypnotics prescriptions & $1.025(0.989-1.063)$ & 0.174 \\
\hline General practitioner visits & $1.014(1.005-1.024)$ & 0.003 \\
\hline General practitioner referrals & $0.989(0.969-1.010)$ & 0.313 \\
\hline Psychotherapy referrals & $1.052(0.977-1.134)$ & 0.181 \\
\hline Laboratory referrals & $0.953(0.883-1.028)$ & 0.209 \\
\hline Psychiatric accident and emergency admissions & $1.066(0.761-1.494)$ & 0.708 \\
\hline Psychiatric non-accident and emergency admissions & $0.927(0.571-1.504)$ & 0.759 \\
\hline General accident and emergency admissions & $1.044(0.954-1.143)$ & 0.352 \\
\hline General non-accident and emergency admissions & $1.036(0.973-1.104)$ & 0.273 \\
\hline \multicolumn{3}{|c|}{ Indicator of Psychiatric Diagnostic Group occurrence in the 180 day prior period } \\
\hline Organic mental disorders and alcohol use disorders & $1.167(0.733-1.856)$ & 0.516 \\
\hline Opioid and other substance use disorders & $0.521(0.240-1.130)$ & 0.099 \\
\hline Schizophrenic disorders & $0.675(0.215-2.120)$ & 0.501 \\
\hline Other psychotic disorders & $0.892(0.169-4.701)$ & 0.893 \\
\hline Bipolar disorder & $0.568(0.111-2.895)$ & 0.496 \\
\hline Major depressions & $1.342(1.187-1.516)$ & $<0.001$ \\
\hline Other specific and atypical affective disorders & $2.936(0.989-8.717)$ & 0.053 \\
\hline Post-traumatic stress disorders & $0.902(0.453-1.796)$ & 0.770 \\
\hline Anxiety disorders & $1.112(0.896-1.379)$ & 0.336 \\
\hline Personality disorders & $2.892(0.923-9.057)$ & 0.068 \\
\hline Impulse control, adjustment disorders, other mental & $0.992(0.780-1.262)$ & 0.948 \\
\hline \multicolumn{3}{|l|}{ Indicator of other medical conditions in the 180 day prior period } \\
\hline Chronic fatigue syndrome & $0.652(0.276-1.536)$ & 0.328 \\
\hline Cancer & $0.983(0.665-1.454)$ & 0.932 \\
\hline Coronary artery disease & $1.010(0.816-1.250)$ & 0.927 \\
\hline Stroke & $0.963(0.675-1.375)$ & 0.835 \\
\hline Diabetes & $0.972(0.717-1.318)$ & 0.857 \\
\hline Fibromyalgia & $0.716(0.379-1.352)$ & 0.303 \\
\hline \multicolumn{3}{|l|}{ General Practice Drug Class Preference } \\
\hline $\begin{array}{l}\text { Frequency of general practice selective serotonin } \\
\text { reuptake inhibitor prescriptions }\end{array}$ & $2.259(1.669-3.058)$ & $<0.001$ \\
\hline
\end{tabular}

However, patients who initiated therapy on a SSRI had an odds ratio over sevenfold higher than patients who initiated therapy on a TCA of having an adequate daily dose and duration of antidepressant therapy consistent with recommended treatment guidelines. In light of the absolute differences between the classes of antidepressants $(6 \%$ for TCA patients and $32.9 \%$ for SSRI patients), this difference is also clinically relevant, particularly when it is considered over a large population

The large sample size and the similarity of findings of previous studies of antidepressant prescribing using these data with other databases in the UK (Donoghue and Tylee, 1996) contributes to the generalizability of these data to the UK as a whole. Consistent with earlier cross-sectional studies (Donoghue and Tylee, 1996; Donoghue et al., 1996; Martin et al., 1997), patients who initiated therapy on SSRIs were more likely to achieve recommended therapeutic doses than their counterparts on TCAs in primary care in the UK. The results were also qualitatively consistent with the earlier longitudinal study in Scotland (MacDonald, 1997). In that study, only $1 \%$ of TCA patients had an adequate dose and duration compared with $29 \%$ of SSRI patients.

In a recent randomized, prospective trial in the Group Health Cooperative Health Maintenance Organization in the USA, patients randomized to desipramine, imipramine or fluoxetine were equally likely to continue on any antidepressant for at least 90 days at an adequate dose (Simon et al. 1996). While that finding contrasts with the results of this study, it may be that the physicians who participated in that trial are systematically different than the typical physicians 
observed in clinical practice in terms of their experience in participating in trials and how they manage patients on antidepressants. Patients referred to that trial may also have been systematically different than patients in the general population. This possibility is consistent with the finding of a similar retrospective (non-clinical trial) study of a broader sample of physicians and patients in the Group Health Cooperative setting which found that the likelihood of continuing antidepressant on any medication was statistically significantly higher for patients who had initiated therapy on the SSRI fluoxetine than patients who had initiated therapy on a TCA (Katon et al., 1992).

The relatively low rates of adequate daily dose and duration for patients initiating therapy on any antidepressant are striking in light of the recent Defeat Depression campaign in the period 1991-96 in the UK (Priest, 1994). For the 1992-94 period of the sample, $5.3 \%$ of TCA patients had an adequate dose and duration of therapy vs $7.9 \%$ of TCA patients in 1995-97, while the figures for SSRI subjects were $29.4 \%$ in 1992-94 and $37.8 \%$ in 1995-97. An earlier cross-sectional analysis of prescriptions in the DIN-LINK data found that the rate of adequate dose of antidepressant prescribing had increased during 1993-95, due largely to increased prescribing of the SSRIs (Donoghue et al., 1996). It would appear that efforts to increase awareness of appropriate treatment strategies with antidepressants have increased the likelihood of achieving adequate daily dose and duration, regardless of which antidepressant they were prescribed. Even in light of this educational awareness program, it would appear that this likelihood is still greater with an SSRI relative to a TCA.

The observed prescribing patterns of TCAs and SSRIs in clinical practice may also have implications for cost-effectiveness comparisons that rely upon controlled clinical trial data. Recent analyses have concluded that because TCAs and SSRIs are generally equally efficacious, TCAs represent a more costeffective solution because of the acquisition price differential (Effective Health Care Bulletin, 1993; Song et al., 1994; Hotopf et al., 1996). This conclusion might have credence if TCAs were used in actual clinical practice in the same way they are used in clinical trials (i.e. at adequate doses for sufficient duration). However, the findings of this and other studies indicate they are not. Rather, patients on TCAs begin at low doses and remain there.

This study's results were found despite the fact that the study design included aspects that favoured the TCAs. First, using an intent-to-treat design allows the results from patients who switched antidepressant class to be attributed to the patient's initial drug class. This favours TCAs because more patients who initiated therapy on TCAs switched to SSRIs than vice versa, and SSRI patients were more likely to achieve adequate daily dose and duration. Second, including lofepramine in the TCA category leads to a higher rate of TCA patients achieving an adequate dose and duration. While lofepramine is a TCA, it is generally thought to have lower toxicity, and thus may be better tolerated, and was found to have a different pattern of prescribing relative to amitriptyline and dothiepin (Donoghue and Tylee, 1996; Donoghue et al., 1996). The lower odds ratio for SSRI patients relative to lofepramine patients may only reflect the greater similarity in the use of SSRIs and lofepramine.
Despite the recommended treatment guidelines in the UK, some have recently argued that lower doses of TCAs are appropriate (Benkert et al., 1996; Moore, 1997; Tan, 1997). A recent meta-analysis of controlled clinical trials also concluded that there is no difference in efficacy rates between the SSRIs and TCAs, irrespective of dosing levels (Canadian Coordinating Office of Health Care Technology, 1998). However, that meta-analysis only considered acute-phase trials (4-12 weeks) and it was not clear in that study what dosage levels of TCAs were considered and in what populations. Thus, it was not possible to draw conclusions regarding the long-term efficacy of TCAs at lower doses for the vast majority of patients who take antidepressants. Achieving all the goals of antidepressant therapy (symptom resolution, restoration of patient functioning and relapse prevention) necessitates considering a long-term perspective beyond acute phase efficacy and treatment. Thus, until more information becomes available regarding the long-term safety and efficacy of low dose TCAs in a controlled clinical trial setting, patients who initiate therapy on SSRIs may maximize the opportunity to receive adequate treatment consistent with guidelines relative to their counterparts on TCAs (Farmer, 1997).

Given the non-randomization into initial antidepressant, it is possible that differences in antidepressant prescribing patterns among patients initiating therapy on one of the antidepressants were the result of unobserved differences across patients or general practitioners that were not controlled for in the analysis. For example, it might be that some patients on the SSRIs differed from TCA patients in underlying severity, which was not captured by the observed variables. However, given the information available in this database and the study design and methods applied, the bias due to unobserved factors is unlikely to be large. For example, to control for baseline patient differences, a 6-month prior period, an extensive set of observable baseline characteristics, and measures of general practices' experience with the study antidepressants were used to control for as many of the baseline patient and physician characteristics as possible. In addition, comorbid disease variables were included to control for observed differences across the patient groups in disease severity. Thus, the observed difference between drug classes is statistically significant even after controlling for these other observed factors.

A recent study (Simon and Fishman, 1998) also found that physicians' habitual prescribing preferences were a stronger determinant of initial antidepressant drug selection than patient characteristics. There is also other evidence indicating that some general practitioners prescribe the same antidepressant for most patients rather than considering specific patient characteristics when selecting the antidepressant (Matthews $e t$ al., 1993). To this end, a variable measuring the frequency of the general practice's prescribing of the SSRIs was included in the DIN-LINK analysis to control for differences in general practices' characteristics, preferences and prior experience with the antidepressants, as was a statistically significant determinant in this study. This variable had not been considered in earlier observational studies of antidepressant use in the UK and was a new variable to bring to this type of analysis.

It is possible that some patients in the study sample did not meet the clinical criteria for major depressive disorder and that 
some patients who were receiving their antidepressant for major depressive disorders were excluded. Variables measuring the type of depression diagnosis on the patients' records were also included to help control for differences in depression severity. Finally, it was not possible to know whether patients were taking their medications. It may be that compliance was worse for TCA patients than SSRI patients if tolerability was a concern for TCA patients. In addition, low dose TCAs may have been used as a hypnotic with some patients, even though a diagnosis of depression was recorded in the database. However, for this to be a significant factor in these results, this effect would have to be large, which is unlikely.

Direct measures of clinical outcomes were not available in these data. Thus, it was not possible to know for certain whether patients who had a dose and duration of antidepressant therapy consistent with guidelines had a greater clinical improvement relative to other patients. However, some evidence exists that demonstrates a link between the pattern of antidepressant prescribing and patient outcome. For example, one study found that 4 months of antidepressant therapy increased measures of work restoration fourfold relative to I month of therapy (Mintz et al., 1992) while another study found that doubling the rate of adequate daily dose and duration led to a significant reduction in major disabilities (Sturm and Wells, 1995). An adequate length of stable antidepressant therapy at an effective dose is also an important determinant of the prevention of relapse (Maj et al., 1992). Additional studies are needed to more directly assess the relationship between treatment consistent with guidelines and clinical outcomes.

It was not possible to identify the reasons for the observed dose and duration of therapy. Potential reasons underlying discontinuation prior to receiving at least 120 days of therapy within the first 6 months may include lack of clinical effect, intolerable adverse events, or spontaneous symptom resolution. It is not possible to determine whether the observed pattern and lengths of therapy were related to clinical symptom resolution or to poor patient or prescriber understanding of the need to continue treatment for a longer period of time. Patients who had shorter prescribing patterns may have been less severely ill and the decision may have been made to discontinue therapy in light of earlier symptom resolution. On the other hand, some of the patients who had less than 120 days within the first 6 months may have been more severely ill but experienced adverse events or lack of efficacy and thus abandoned treatment altogether.

This study has shown differences in the patterns of antidepressant prescribing among the most common antidepressants in primary care in the UK. It would be useful to study the relationships between patterns of antidepressant prescribing, health care expenditures, and clinical outcomes observed within this primary care setting in the UK. Finally, it would be useful to consider whether patterns of antidepressant prescribing differ among the most common SSRIs.

\section{Acknowledgments}

This study was funded by Lilly Research Laboratories, Eli Lilly and Company, Indianapolis, IN, USA. Nicky Richards and Mark
Henwick of CompuFile, Woking, UK, provided valuable technical assistance.

\section{Address for correspondence}

Timothy R. Hylan

Research Scientist

Lilly Research Laboratories (drop code 2646)

Lilly Corporate Centre

Eli Lilly and Company

Indianapolis, IN 46285

USA

Email: hylant@lilly.com

\section{References}

Agency for Health Care Policy Research (1993) Treatment of major depression: clinical practice guidelines. AHCPR Publication 93-0550;1(5)

Anderson I M, Tomenson B M (1994) The efficacy of selective serotonin reuptake inhibitors in depression: a meta-analysis of studies against tricyclic antidepressants. J Psychopharmacol 8: 238-249

Ashcraft MLF, Fries BE, Nerenz D R, Falcon SP, Srivastava SV, Lee CZ (1989) A psychiatric patient classification system: an alternative to diagnosis-related groups. Med Care 27: 543-557

Benkert O, Szegedi A, Wetzel H (1996) Minimum effective dose for antidepressants - an obligatory requirement for antidepressant drug evaluation? Int Clin Psychopharmacol 11: 177-185

British Association for Psychopharmacology (1993) Guidelines for treating depressive illness with antidepressants. J Psychopharmacol 7:19-23

Canadian Coordinating Office of Health Care Technology Assessment (1998) A clinical and economic evaluation of selective serotonin reuptake inhibitors in major depression. Ontario, Canada

Donoghue J M (1995) A comparison of prescribing patterns of selective serotonin reuptake inhibitors in the treatment of depression in primary care in the United Kingdom. J Serotonin Res 1: 47-51

Donoghue JM (1996) Prescribing patterns of selective serotonin reuptake inhibitors in primary care: a naturalistic follow-up study. J Serotonin Res 4: 267-270

Donoghue JM, Tylee A (1996) The treatment of depression: prescribing patterns of antidepressants in primary care in the UK. Br J Psychiatry 168: 164-168

Donoghue J M, Tylee A, Wildgust H (1996) Cross-sectional database analysis of antidepressant prescribing in general practice in the United Kingdom. BMJ 313: 811-812

Effective Health Care Bulletin (1993) The treatment of depression in primary care. Effective Health Care Bull 5: 1-12

Fairman K (1997) The doctor told me to cut the pills in half: practical considerations in using claims databases for outcomes research. Drug Benefit Trends 9: 30-36

Farmer R (1997) Managing depression: a matter of record. Primary Care Psychiatry 3 (Suppl. 1): S11-S16

Henry JA (1995) Relative mortality from overdose of antidepressants. BMJ 310: 221-224

Hotopf M, Lewis G, Normand C (1996) Are SSRIs a cost-effective alternative to tricyclics? Br J Psychiatry 168: 404-409

Katon W, Von Korff M, Lin E, Bush T, Ormel J (1992) Adequacy and duration of antidepressant treatment in primary care. Med Care 30: $67-76$

MacDonald T M (1997) Treatment of depression: prescription for success? Primary Care Psychiatry 3 (Suppl. 1): S7-S10 
Maj M, Veltro F, Pirozzi R, Lobrace S, Magliano L (1992) Pattern of recurrence of illness after recovery from an episode of major depression: a prospective study. Am J Psychiatry 149: 795-800

Martin R M, Hilton S R, Kerry S M, Richards N M (1997) General practitioners' perceptions of the tolerability of antidepressant drugs: a comparison of selective serotonin reuptake inhibitors and tricyclic antidepressants. BMJ 314: 646-651

Matthews K, Eagles J M, Matthews CA (1993) The use of antidepressant drugs in general practice. Eur J Clin Pharmacol 45: 205-210

Mintz J, Mintz LI, Arruda M J, Hwang S S (1992) Treatments of depression and the functional capacity to work. Arch Gen Psychiatry 49: 761-768

Moore MV (1997) More on what is an effective dose [letter]. BMJ 314: 827

Montgomery SA (1994) Selective serotonin reuptake inhibitors: meta-analysis of discontinuation rates. Int Clin Psychopharmacol 9: 47-53

Priest RG (1994) Improving the management and knowledge of depression. Br J Psychiatry 164: 285-287
Royal College of Psychiatrists (1992) Recognition and management of depression in general practice. BMJ 205: 1198-1202

Simon G E, Fishman P (1998) Cost implications of initial antidepressant selection in primary care. Pharmacoeconomics 13:61-70

Simon G E, Von Korff M, Wagner E H, Barlow W (1993) Patterns of antidepressant use in community practice. Gen Hosp Psychiatry 15: $399-408$

Simon G E, Von Korff M, Heiligenstein J H, Revicki DA, Grothaus $\mathrm{L}$, Katon W et al. (1996) Initial antidepressant choice in primary care: effectiveness and cost of fluoxetine vs. tricyclic antidepressants. JAMA 275: 1897-1902

Song F, Freemantle N, Sheldon TA (1994) Selective serotonin reuptake inhibitors: meta-analysis of efficacy and acceptability. BMJ 306: 683-687

Sturm R, Wells K (1995) How can care for depression become more cost-effective? JAMA 273: 51-58

Tan RS (1997) Low dose tricyclic antidepressants are effective in treating major depression [letter]. BMJ 314: 827

World Health Organization Mental Health Collaborating Centers (1989) Pharmacotherapy of depressive disorders: WHO consensus statement. J Affect Disord 17: 197-198 